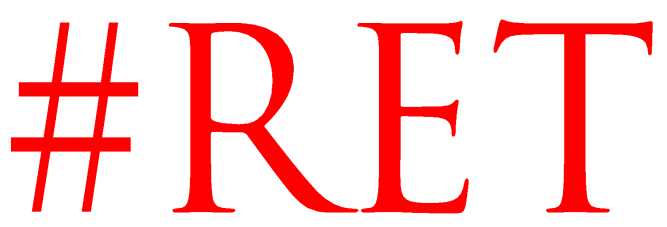

Revista Española de la Transparencia

Núm. 10. Primer Semestre 2020

ISSN 2444-2607. Págs. 151-175

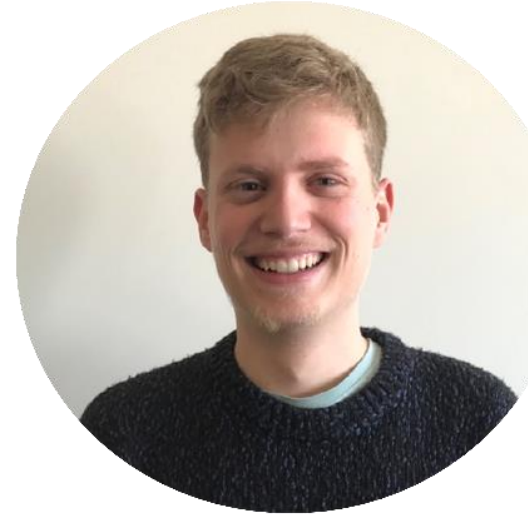

Carlos José Aguilar Garcia1

Universidad de Granada. España

\title{
La transparencia en las páginas web de los parlamentos según el tipo de régimen: el caso del Magreb
}

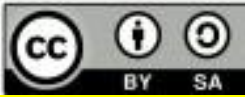

RECIBIDO: 4 de diciembre de 2019 ACEPTADO: 30 de enero de 2020

RESUMEN: La transparencia online y las nuevas tecnologias están en el orden del día en la actualidad. Sin embargo, son pocos los estudios que se centren en la transparencia online en regímenes que no sean democráticos. Por ello, en el presente artículo trataremos de estudiar la transparencia en las páginas web de los parlamentos dependiendo del tipo de régimen al que pertenecen. Para ello utilizaremos el caso del Magreb, desarrollando un listado de variables a través del ejemplo del Senado español. Estudiaremos estas variables, en los tipos de transparencia que representan, y analizaremos cuáles y cuáles no aparecen en Argelia, Túnez y Marruecos, para conocer así si el tipo de régimen político influencia sobre la transparencia online de sus parlamentos

PALABRAS CLAVE: Transparencia, Magreb, Regímenes híbridos, parlamentos, legitimidad.

CONTENIDOS: 1. Introducción. - 2. Marco teórico. - 3. Metodología. - 4. Trasparencia activa: la actualidad parlamentaria. - 5. Transparencia pasiva: composición y organización. - 6. Transparencia colaborativa. - 7. Conclusiones. - Bibliografía.

\footnotetext{
${ }^{1}$ Actualmente es becario de colaboración del departamento de Ciencia Política y de la Administración de la Universidad de Granada. Su interés investigador se centra en la Teoría Política y la Historia de las Ideas, siendo autor de distintas comunicaciones sobre dicha temática.
} 


\section{Carlos José Aguilar García}

\section{Transparency in the web pages of parliaments according to the type of regime, the case of the Maghreb}

ABSTRACT: Online transparency and new technologies are on the agenda today. However, there are few studies that focus on online transparency in nondemocratic or Hybrid regimes. Therefore, in this article we will try to study transparency in the web pages of parliaments depending on the type of regime to which they belong. For this we will use the case of the Maghreb, developing a list of variables through the example of the Spanish Senate. We will study these variables, in the types of transparency they represent, and we will analyze which and which do not appear in Algeria, Tunisia and Morocco, in order to know if the type of political regime influences the online transparency of their parliaments.

KEYWORDS: Transparency, Maghreb, Hybrid Regimes, parliaments, legitimacy. 


\section{La transparencia en las páginas web de los parlamentos ...}

\section{INTRODUCCIÓN.}

La transparencia es un elemento fundamental para el desarrollo democrático de los regímenes políticos. El contacto de la administración con el ciudadano facilita el alcance de la información y la rendición de cuentas. En un momento en el que el desarrollo de las tecnologias hace cada vez más sencillo el acceso a todo tipo de información, es importante abordar, desde el ámbito académico, la situación de la transparencia en la red de diferentes regímenes.

Con frecuencia, cuando nos referimos a la e-administración y su capacidad de llevarnos a un gobierno abierto, de carácter democrático y que rinda constantemente cuentas ante sus ciudadanos, solo miramos a los regímenes occidentales o a las democracias consolidadas. De esta manera, tenemos mucha bibliografía sobre la transparencia en la red de la Administración de países como España, pero no sucede lo mismo respecto de países con otras realidades políticas, lejanas a las democracias. Ante esto, este trabajo pretende aportar algo de nitidez a los estudios sobre transparencia en internet de países no democráticos. Para ello, el trabajo se centrará en el estudio de la transparencia de las páginas web de los parlamentos de diferentes países del Magreb (Argelia, Túnez y Marruecos), en comparación con España.

Estas páginas web se analizarán de manera sincrónica, en un mismo periodo de tiempo, siendo extraídos los datos a lo largo del mes de octubre de 2019. Luego, en el caso de que estas sufran una modificación, en los próximos meses, sería necesaria una revisión del trabajo, lo que permitiría analizar si hay avances o retrocesos en materia de transparencia.

Las dimensiones con las que analizaremos la transparencia en sus páginas web serán cuatro, extraídas de manera comparada con lo que muestra la página web del Senado de España, un país democrático y que avanza cada vez más en la transparencia (como podemos observar en la evolución de España en el índice Corruption Perception Index, de Transparencia Internacional, donde ha pasado de ser el país número 41 de 176 en 2016, al país en el puesto 30 de 180 países con respecto al nivel de transparencia, obteniendo una puntuación de 62 sobre 100, en vez de la de 58 que tenía en 2016 y 2018)². Estas dimensiones son: la actividad parlamentaria, que se subdividirá a su vez en nueve variables; otra dimensión de organización y composición, subdividida en cuatro dimensiones menores, que serán: los senadores o parlamentarios con 11 variables; los grupos parlamentarios con 10 variables; la administración parlamentaria con dos variables; y los órganos con 3. Además, encontramos las dimensiones de datos culturales y búsqueda temática.

\footnotetext{
2 Para más información pueden consultar los informes de Transparencia Internacional de 2016, 2018 y 2019 en: https://www.transparency.org/country/ESP
} 


\section{Carlos José Aguilar García}

Estas dimensiones las utilizaremos durante el trabajo para intentar validar nuestra hipótesis, que afirma que existen diferencias significativas en la transparencia de los portales de los parlamentos según el tipo de régimen político. Esperamos encontrar mayores cotas de transparencia en los regímenes democráticos, y los que más se aproximan a las democracias, antes que en los autoritarios. Concretamente, además, queremos conocer cada una de las dimensiones en las diferentes realidades de los países magrebies seleccionados, estudiando si existe más transparencia en la actividad parlamentaria o de la vida de sus políticos, o si existen apartados en sus páginas web que se refieran a la dimensión cultural.

Este trabajo es de interés académico ya que, como señalamos al inicio, los estudios relacionados con la transparencia y la e-administración no se suelen centrar en el Magreb, sino que por lo general se fijan en los paises occidentales y en los regímenes democráticos. El trabajo nos puede servir para conocer hasta qué punto existen diferencias entre los tipos de regímenes y su trato de la información en internet, y si esto es un reflejo en los principales índices. A nivel personal, el estudio se motiva por la intención de combinar los conocimientos adquiridos sobre dos áreas diferentes de la Ciencia Política, por un lado, las Administraciones Pública en su relación con la gestión pública y, por otro, los estudios de área sobre el Magreb.

Por último, destacaremos que el trabajo se estructurará de la siguiente manera: en primer lugar, se expondrá el marco teórico y la metodología que aplicaremos a esta investigación, y a continuación, mostraremos los resultados obtenidos en el estudio de las diferentes dimensiones, dedicándoles un apartado a cada una de las dos primeras y tratando conjuntamente las dos últimas, para finalmente cerrar el trabajo con una conclusión con los datos obtenidos.

\section{MARCO TEÓRICO.}

Los elementos teóricos que vamos a utilizar en este trabajo van a estar vinculados fundamentalmente al estudio de los tipos de regímenes y a la transparencia en su relación con la e-administración. Centrarnos en ellos será la clave para suplir las carencias que tenemos a la hora de abordar este trabajo por la inexistencia de estudios que se centren en la transparencia de los portales de internet en el Magreb o en regímenes no democráticos. Para ello, en el marco teórico nos centraremos en primer lugar en analizar los estudios sobre tipos de regímenes en el Magreb, y, en segundo lugar, en la transparencia en los portales digitales. Combinando ambas, veremos si existe algún tipo de correlación entre el avance del e-goverment y los tipos de regímenes. Para conectar estas dos grandes áreas de la Ciencia Política utilizaremos estudios sobre consolidación democrática, autoritarismo, legitimidad y parlamentos.

Con la finalidad de acotar el número de regímenes a estudiar, decidimos seleccionar unos cuantos que nos permitieran cubrir un espectro más o menos considerable, desde una democracia defectiva, hasta un autoritarismo cerrado 


\section{La transparencia en las páginas web de los parlamentos...}

pasando por uno competitivo. Es por ello por lo que, en primer lugar y para vislumbrar cuantitativamente los regimenes de los que disponemos, estudiamos los diferentes índices de democracia en el Norte de África con esta gráfica:

\section{Gráfica 1: índices de democracia y transparencia en los paises del Norte de África}

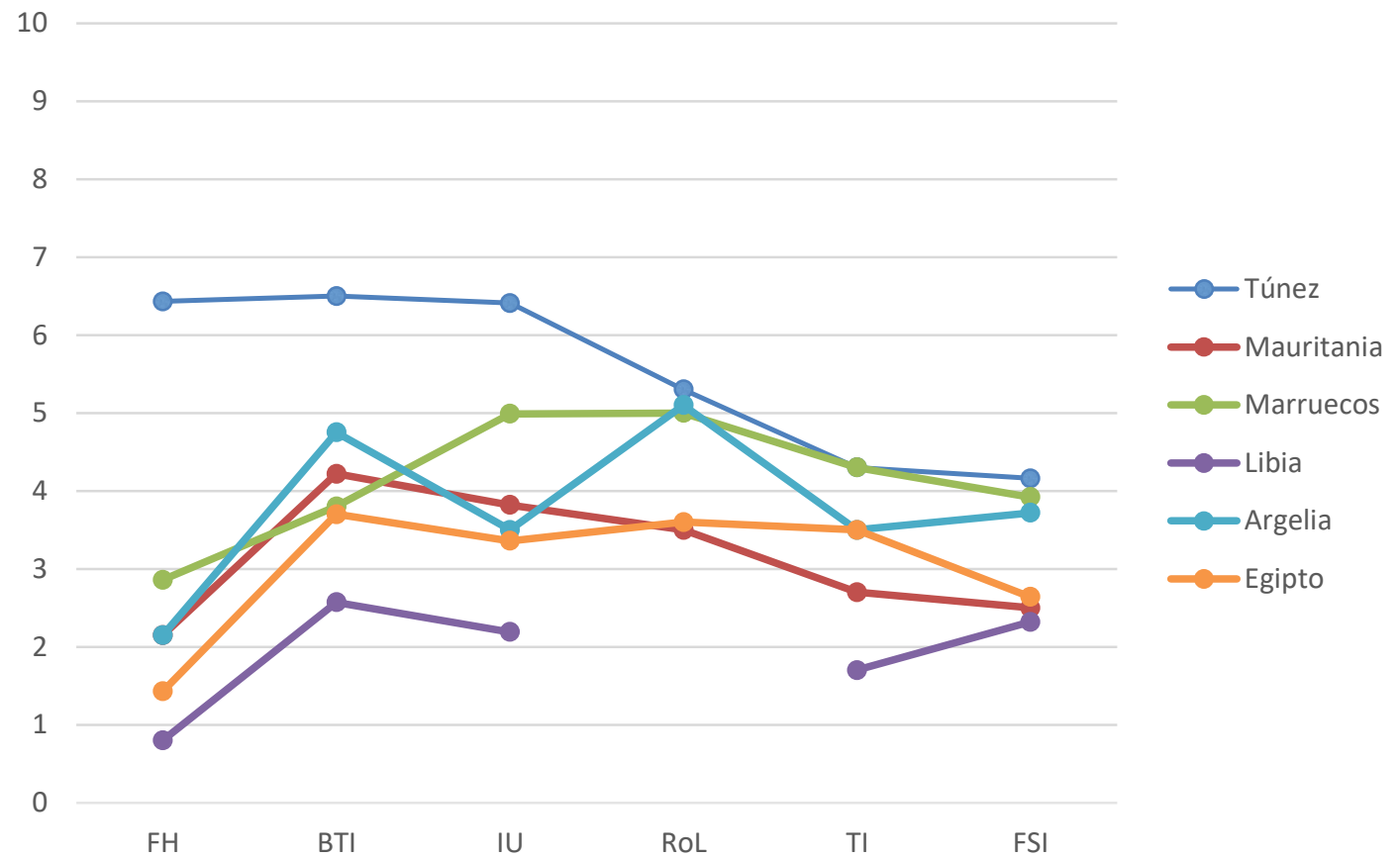

Fuente: elaboración propia a partir de los principales indices de democracia y transparencia ${ }^{3}$.

Esta gráfica, cuya realización explicamos en la metodología, nos revela que existen diferencias considerables entre todos los paises y Túnez, como mejor opción, y

3 Los indices incluidos en la gráfica son:

FH: Freedom House (2018): En Internet: https://freedomhouse.org/report/freedom-world/freedomworld-2018

IU: The Economist Intelligence Unit (2017): En Internet: https://infographics.economist.com/2019/Democracylndex/

FSI: Fragile State Index (2019): En Internet: https://fragilestatesindex.org/category/analysis/fragilestates-index-2019/

Tl: Transparency International, Corruption Perception Index (2018): En Internet: https://www.transparency.org/cpi2018

BTI: Bertelsmann Transformation Index (2018): En Internet: https://www.bti-project.org/en/countryreports/

RoL: Rule Of Law Index (2019): En internet: https://worldjusticeproject.org/ourwork/publications/rule-law-index-reports/wjp-rule-law-index-2019 


\section{Carlos José Aguilar García}

Libia como peor. En el resto, Mauritania, Marruecos, Argelia y Egipto, existe una mayor similitud, a pesar de sus diferentes regímenes. Sin embargo, hemos decidido seleccionar los casos de Túnez, Argelia y Marruecos, debido a que los tres son regímenes catalogados de forma diferente pero que en el Transparency Index toman valores muy cercanos. Además, hemos descartado Egipto por no pertenecer al Magreb, Libia por su débil situación política y Mauritania, ya que creemos que Argelia cubre parte de su espectro.

Una vez establecidos los países que vamos a estudiar, es el momento de conocer las categorías de regímenes que existen para poder situarlos. Una de las grandes divisiones que debemos plantearnos es qué tipos de regímenes hay además de la democracia. En la academia está bastante asentada la existencia de regímenes híbridos (Szmolka, 2010), pseudo democracias (Diamond, Linz, Lipset, 1995) o democracias iliberales (O'Donnell y Schmitter, 1994), pero la cuestión que primeramente queremos abordar es cuál es el elemento contrario del eje democracia y no democracia que vamos a utilizar para nuestro estudio, si el autoritarismo o el totalitarismo.

Es probable que el concepto más antagónico con la democracia sea el de totalitarismo, pero como recogió Hannah Arendt (2006), los principales regímenes totalitarios fueron el nacismo alemán y el stalinismo soviético. Es asi que Linz (1990: 10) nos señala que: "Si se usa una definición estricta del totalitarismo, se aplicaría a los gobiernos de Hitler, de Stalin y de otros regímenes comunistas, pero no a todos ellos y probablemente ni siquiera a la Italia de Mussolini". Juan Francisco Fuentes nos dirá a su vez que el concepto de totalitarismo ha sufrido una evolución que le ha llevado a "una pérdida paulatina de protagonismo tras la muerte de Stalin y el comienzo del deshielo Este/Oeste" (Fuentes, 2006:195). Los planteamientos de estos autores evidencian la lejanía de ese concepto en la actualidad, ya que si bien algunos países gozan de características totalitarias, el término no tiene una capacidad explicativa coherente, o al menos tan amplia como el de autoritarismo.

El autoritarismo y la democracia serán nuestras dos categorias que nos permitirán cubrir todo el espectro de regímenes que aqui nos competen. Los regímenes híbridos, ahora, podrán ser definidos en uno u otro sentido, puesto que como señala Szmolka, la forma de utilizar esta terminología implica un movimiento hacia la democracia o hacia el autoritarismo, por lo que debemos tener en cuenta si adjetivamos democracias o autoritarismos (Szmolka: 2010, 105)

Szmolka realizará un extenso trabajo en 2010 con la finalidad de desarrollar una terminología de regímenes hibridos, en la que nosotros nos basaremos. Esta defenderá que existen democracias plenas, democracias defectivas de carácter tutelado o democracias defectivas iliberales, autoritarismos pluralistas cuasi competitivos o hegemónicos restrictivos y autoritarismos cerrados (Szmolka, 2010: 117-119). 


\section{La transparencia en las páginas web de los parlamentos ...}

Al año siguiente, la misma autora desarrolló un trabajo en el que intentaría clasificar a los países árabes dentro de esas categorias. Con un extenso aparataje metodológico concluyó que Marruecos era un autoritarismo pluralista cuasi competitivo, mientras que Argelia era un autoritarismo hegemónico restrictivo. El caso de Túnez es particular, ya que ella lo catalogaba en aquel momento de autoritarismo hegemónico restrictivo, debido a elementos como (Szmolka, 2011:22):

"La competencia se encuentra limitada por la posición hegemónica o ultradominante de un determinado partido, grupo o coalición en los procesos políticos, por las trabas a la actividad de oposición o por las irregularidades cometidas en los procesos electorales. De esta forma, se permite la interacción de los partidos en el sistema político, pero no la competencia en el acceso al poder. Las elecciones sirven principalmente para formar mayorías parlamentarias de apoyo al gobierno".

Esta categoría es comprensible en el momento en el que Szmolka realizó su trabajo. En la actualidad, con la evolución de Túnez y lo que señalan índices como Freedom House o BTI, deberíamos catalogar a Túnez como una democracia defectiva. Es más, investigando nuevos trabajos de la autora, en 2019, en su obra "Una propuesta de tipología de sistemas de partidos para regímenes democráticos y autoritarios pluralistas. Su aplicación a los países del Magreb", recoge que Túnez es una democracia defectiva, Marruecos un autoritarismo cuasi competitivo y Argelia un autoritarismo hegemónico (Szmolka, 2019:93).

Los elementos de estas categorías que más nos pueden interesar para nuestro estudio son: que las democracias defectivas, como regímenes político que no han culminado su proceso de consolidación democrática, "ven limitados su desarrollo por distintos factores: funcionamiento inadecuado en los frenos y contrapesos de las instituciones representativas, fenómenos de corrupción y clientelismo" (Szmolka, 2011,16). Mientras que los regímenes autoritarios (que serán hegemónicos si su grado de competencia política es menor, y cuasi competitivos si es mayor) destacan por la "falta de autonomía de las instituciones, la falta de equilibrio o contrapesos entre poderes 0 la presencia de actores influyentes sin responsabilidad política o por actores formales que centralizan el proceso institucional y político en detrimento de las instituciones representativas" (Szmolka, 2011:16).

De esta manera, y siguiendo los postulados de Szmolka, tenemos para comparar un régimen democrático defectivo, un autoritarismo pluralista cuasi competitivo y un autoritarismo hegemónico restrictivo, en su transparencia en los portales online de sus parlamentos.

Esta variedad de regímenes debemos compararlos según sus parlamentos por diferentes cuestiones. Entre ellas, porque las instituciones de poder de los regímenes juegan un papel fundamental en su desarrollo, sean democráticos o no los parlamentos van a ser un elemento de transparencia y legitimidad, pese a que 


\section{Carlos José Aguilar García}

en los regimenes democráticos tengan una importancia real y unas funciones de contrapeso, y en los híbridos en ocasiones solo cubran una función formal.

La legitimidad, para afirmar la importancia de esta función representada en los parlamentos, ha de entenderse como "la creencia en que, a pesar de las deficiencias y los fallos, las instituciones politicas existentes son mejores que otras que hubieran podido establecerse" (Montero y Morlino, 1993:10). Este elemento ha sido un problema constante en el Magreb, donde "el problema de la legitimidad es consustancial con la formación de los estados territoriales magrebies" (Camau, 1992:88), y con la mayoría de los gobiernos autoritarios del mundo árabe, que se sustentan en su propia robustez (Entelis, 2008:39). Linz nos dirá que uno de los factores de crisis en los regímenes autoritarios es su falta de eficiencia y legitimidad (1990:13), dos elementos que incorporan los parlamentos y que son visibles en las cuestiones que le muestran a la ciudadanía. Como señala Yolanda Fernández (2008: 77):

Algunos de los aspectos en los que el Sector Público es esencial son la mejora de las infraestructuras de comunicación, la preparación básica del capital humano, la agilidad en la toma de decisiones y la eficacia y eficiencia en la prestación de los servicios y en su gestión.

La eficiencia y la eficacia, relacionadas con la transparencia, aportan legitimidad a los sistemas. El estudio de la transparencia de las páginas web de los parlamentos de estos regímenes nos permitirá ver qué le muestra a los ciudadanos el parlamento de esos países, conociendo de esta manera si utiliza la transparencia como un elemento de legitimación por la eficiencia o la eficacia, ya sea ocultando elementos que no quiere dar a conocer o publicando de manera selectiva lo que le es beneficioso. Como nos señala María Angustias Parejo, "en la evolución histórica de la legitimidad en el Magreb, las elecciones como fuente de legitimidad política adquieren un renovado protagonismo" (16:2010), lo que incluye en sí a los parlamentos. Esto es fruto de la necesidad de los regímenes autoritarios de poner en marcha ciertas medidas de liberalización para reducir a la oposición (Linz, 1990:14), y una de las principales son los parlamentos.

Esta conflictividad con la legitimidad es lo que nos obliga a enfocar la transparencia de estos regímenes desde la teoría de la legitimidad. Mientras que la teoría neoinstitucional "se configura como una teoría que va a abordar un estudio sociológico de las instituciones, entendidas estas como los espacios donde los diferentes actores sociales desenvuelven sus prácticas" (Curto-Rodríguez, 2019:181), y la teoría de la agencia se centra en la delegación de funciones de ciudadanos al gobierno, la teoría de la legitimidad, hace referencia "a la capacidad de generar obediencia sin tener que recurrir a la coacción" (Curto-Rodríguez, 2019:181), convierte a la transparencia en un instrumento para "recuperar la confianza perdida del ciudadano" (Curto-Rodríguez y García-García, 2019:4). 


\section{La transparencia en las páginas web de los parlamentos ...}

Evidentemente no podemos aplicar un enfoque neoinstitucional en regímenes en los cuales las instituciones no incorporan una pluralidad de actores, sino más bien pequeños grupos de poder que controlan el pais de forma patrimonial (Entelis, 2008:43), ni hacer referencia a la teoria de la agencia, ya que las funciones ejercidas por el poder no son recibidas a través de una delegación sino de una extracción forzosa. En definitiva, es la teoría de la legitimidad la que cobra más sentido en el estudio de estos regímenes, que como señalamos con anterioridad, han estado históricamente en su búsqueda.

Por otra parte, los parlamentos tienen un vínculo esencial con la democracia, luego estudiarlos en su dimensión de transparencia según el tipo de régimen nos aporta una visión de la relación de los regímenes hibridos con un elemento fundamental de la democracia. De esta manera, José Tudela nos dirá que "junto a la necesaria visión del Parlamento como derecho, de los ciudadanos, resulta forzoso preguntarse por la presencia de los derechos y libertades en el quehacer cotidiano de la institución" (2012:216). Con ello vemos como de los parlamentos no sólo debemos observar hacia el exterior en su reconocimiento de derechos, sino que también hacia dentro en su calidad democrática, donde entra la transparencia. De lo contrario estaríamos cayendo en el error de concebir el parlamento sólo como una herramienta de la democracia republicana, que se estructura en las dimensiones normativas de representación, participación, inclusión y deliberación (Maíz, 15:2006). Cuando trabajamos con regímenes hibridos tenemos que tener una comprensión más laxa de los parlamentos y no concebirlos de manera normativa.

En definitiva, debemos conectar estas dos áreas de la Ciencia Política porque los parlamentos, la legitimidad y la transparencia juegan un rol fundamental y común en la transformación o el mantenimiento de los regímenes. Según Morlino, "un régimen autoritario que ha sido capaz de llevar a cabo políticas de socialización y de supresión eficaces puede dejar una sociedad civil mal organizada, fragmentada, débil y pasiva" (2015:22), esta sociedad quedará configurada por la forma de ejercerse el poder en el régimen para futuros cambios. Como señala Morlino es diferente la institucionalización y la consolidación de la democracia (1986:17), la consolidación requiere de varios factores, dentro de los cuales destaca la estructura del régimen (Liebert, 1988: 101), estando la institucionalización dentro de la consolidación. La democracia de esta manera, quedará instaurada "cuando las principales estructuras del régimen estén preparadas" (Morlino, 2015: 24).

Con todo lo anteriormente señalado podemos concluir que el estudio de los parlamentos es un elemento central para conocer las diferencias de los regímenes, que usarán estos para legitimarse, debilitar a la oposición o abordar transiciones entre otros. El estudio de la transparencia electrónica de esos parlamentos se vuelve fundamental en este momento, en el cual no hemos encontrado investigaciones sobre esta materia y sabemos que es una vía útil para la legitimación de los regímenes. Aun así, seguiremos planteando como hipótesis que los regímenes serán más transparentes en sus páginas web cuanto más 


\section{Carlos José Aguilar García}

democráticos sean, ya que tendrán menos información que ocultar a sus ciudadanos.

Con respecto a la transparencia es fundamental recoger los planteamientos de Manuel Villoria, quien en 2018 desarrolló en su artículo "La transparencia, la imparcialidad y la calidad de la democracia", que tras la tercera ola democratizadora se pensaba que la democracia era imparable, pero que con el fracaso de la primavera árabe y el reflujo autoritario es el momento de repensar esa realidad (Villoria, 2018: 1). Según él, la respuesta al ataque a la democracia debía ser "más y mejor democracia", esto dependía de un sistema de controles que evite las derivas autoritarias. La mejor forma en el siglo XXI de garantizar esos controles es el gobierno electrónico, que ha de aspirar a producir democracias de calidad con rendición de cuentas vertical y horizontal, pudiendo la ciudadanía evaluar la gestión del gobierno y aportando además de la legitimidad política una legitimidad desde los resultados (Villoria, 2019:8).

La noción de transparencia, como señalan Jesús García y Ricardo Curto (2018:1052), está lejos de ser clara, se la ha definido como "un proceso en constante renovación que los Gobiernos deben liderar" (Delgado, 2015:28) y que se fundamenta en el derecho de acceso a la información y como "pilar fundamental del Gobierno Abierto y el Buen Gobierno" (Delgado, 2015:27). Pero como señalan Canales y Romero (2017:115): "el modelo existente de sistema político implicará un determinado modelo de Estado, de Gobierno y por ende, de Administración Pública", afectando evidentemente a la concepción de transparencia. Esta última, como un elemento que sobrepasa la "información, la comunicación y la documentación administrativa claras y fiables" que implicando también facilidad de acceso y receptividad (Canales y Romero, 2017:116), puede estudiarse según los tipos de régimen, para conocer en qué grado se materializa conforme al grado de asentamiento democrático (desde la democracia defectiva, hasta el autoritarismo cuasi competitivo hasta el autoritarismo hegemónico).

Son múltiples las propiedades beneficiosas que se le atribuyen a la transparencia, siendo una vía para frenar la corrupción o las arbitrariedades (García-García y Curto-Rodríguez, 2018:1052), con independencia del tipo de régimen. La combinación de una cultura administrativa con sistemas de controles y equilibrios basados en una mayor información es el mejor freno de la corrupción (Cerillo, 2011:277). La transparencia, por ende, se define como un elemento contrario a la corrupción, y la corrupción va de la mano del autoritarismo, como nos señalan los índices recogidos en la gráfica del inicio (Transparency Index), donde podemos observar que los regímenes menos democráticos son los que tienen menor nivel de transparencia y mayores cotas de corrupción (tomando Túnez (democracia defectiva) y Marruecos (autoritarismo cuasicompetitivo) las mejores puntuaciones, y a continuación los regímenes autoritarios hegemónicos). 


\section{La transparencia en las páginas web de los parlamentos ...}

La transparencia, según Cerrillo, tiene tres dimensiones: una transparencia activa, que se encarga de la difusión de la información del sector público; una transparencia pasiva que permite el acceso a la información del sector público y una transparencia colaborativa relacionada con la reutilización de la información del sector público. Esta última se centra en que los propios ciudadanos se conviertan en generadores de información mediante las nuevas tecnologías (Cerillo, 2011: 287-292).

En nuestro trabajo nos centraremos en el estudio de la transparencia activa, pasiva y colaborativa recogida en las páginas web de los senados marroquí y argelino y el congreso tunecino, tomando como referencia tres de las ideas del gobierno abierto que plantea Villoria: el gobierno transparente y la rendición de cuentas, el gobierno participativo y promotor de civismo y el gobierno eficiente, colaborador y generador de conocimiento (Villoria, 2015: 69-101). Traduciremos estas ideas en cuatro dimensiones prácticas: la actividad parlamentaria, la composición y organización, la búsqueda libre y los apartados de cultura.

En definitiva, a raíz de este marco, este trabajo intentará esclarecer con las variables definidas a continuación, si existe una relación entre la transparencia en las páginas web de los parlamentos y el tipo de regímenes que los albergan.

\section{METODOLOGÍA}

Antes de ahondar en la metodología sobre el cuerpo de nuestro trabajo, es conveniente señalar la aplicada en ciertos rasgos de nuestro marco teórico. Como se puede observar en el marco teórico, hemos realizado una gráfica con los distintos índices que evalúan las cualidades de los regímenes, desde indices de transparencia hasta índices de democracia, que explicaremos en ese apartado, sobre los países del Norte de África. Sin embargo, lo que aquí nos compete es conocer cómo hemos elaborado esas gráficas. En primer lugar, hemos de señalar que esta gráfica ha sido generada con datos secundarios y cuantitativos obtenidos de los principales índices, que recogemos en las siguientes tablas. 


\section{Carlos José Aguilar García}

Tabla 1. Índices de democracia y transparencia

\begin{tabular}{|l|c|c|c|c|c|c|}
\hline Pais/Indice & $\begin{array}{c}\text { Freedom } \\
\text { House (7) }\end{array}$ & BTI (10) & $\begin{array}{c}\text { The } \\
\text { economist } \\
\text { Intelligence } \\
\text { Unit (10) }\end{array}$ & $\begin{array}{c}\text { Rule of Law } \\
\text { (1) }\end{array}$ & $\begin{array}{c}\text { Transparency } \\
\text { International } \\
(100)\end{array}$ & $\begin{array}{c}\text { Fragile State } \\
\text { Index (120) }\end{array}$ \\
\hline Túnez & 2,5 & 6,5 & 6,41 & 0,53 & 43 & 70,1 \\
\hline Mauritania & 5,5 & 4,22 & 3,82 & 0,35 & 27 & 90,1 \\
\hline Marruecos & 5 & 3,8 & 4,99 & 0,5 & 43 & 73 \\
\hline Libia & 6,5 & 2,57 & 2,19 & - & 17 & 92,2 \\
\hline Argelia & 5,5 & 4,75 & 3,5 & 0,51 & 35 & 75,4 \\
\hline Egipto & 6 & 3,7 & 3,36 & 0,36 & 35 & 88,4 \\
\hline
\end{tabular}

\begin{tabular}{|l|c|c|c|c|c|c|}
\hline $\begin{array}{c}\text { Freedom } \\
\text { House/ Pais }\end{array}$ & Túnez & Mauritania & Marruecos & Libia & Argelia & Egipto \\
\hline Do Políticos & 2 & 6 & 5 & 7 & 6 & 6 \\
\hline $\begin{array}{l}\text { Libertades } \\
\text { civiles }\end{array}$ & 3 & 5 & 5 & 6 & 5 & 6 \\
\hline
\end{tabular}

Fuente: Elaboración propia a través de los principales índices de democracia.

Como podemos observar, los índices recogidos están en diferentes bases numéricas, y no todos tienen una relación creciente. Es decir, no en todos una mejora de la calidad democrática supone más puntos, por ejemplo, Freedom House. Es por ello por lo que hemos realizado otra tabla, invirtiendo los valores que decrecían con la democracia, y convirtiendo todos los índices en base decimal, a partir de la cual hemos realizado la gráfica del marco teórico (es decir, si Fragile State Index, por ejemplo, tiene una relación decreciente con la transparencia y no en base decimal, hemos realizado una regla de tres para convertirlo a base decimal y luego hemos restado el valor a 10, para obtener el valor en base decimal ascendente). Debemos destacar que en esta tabla hemos colocado entre paréntesis los valores de los índices que decrecian con el aumento de las cualidades positivas si los hubiésemos considerado invertidos en base decimal. 


\section{La transparencia en las páginas web de los parlamentos ...}

\section{Tabla.2 Índices de democracia y transparencia en base decimal y de manera creciente}

\begin{tabular}{|c|c|c|c|c|c|c|}
\hline $\begin{array}{l}\text { Pais/Índices } \\
\text { sobre } 10\end{array}$ & $\begin{array}{l}\text { Freedom } \\
\text { House }\end{array}$ & BTI & $\begin{array}{c}\text { The } \\
\text { economist } \\
\text { Intelligence } \\
\text { Unit }\end{array}$ & Rule of Law & $\begin{array}{c}\text { Transparency } \\
\text { International }\end{array}$ & $\begin{array}{l}\text { Fragile State } \\
\text { Index }\end{array}$ \\
\hline Túnez & $\begin{array}{c}6,43 \\
(-3,57)\end{array}$ & 6,5 & 6,41 & 5.3 & 4,3 & $\begin{array}{c}4,16 \\
(-5,84)\end{array}$ \\
\hline Mauritania & $2,15(-7,85$ & 4,22 & 3,82 & 3,5 & 2,7 & $2,5(-7,5)$ \\
\hline Marruecos & $\begin{array}{c}2,86 \\
(-7,14) \\
\end{array}$ & 3,8 & 4,99 & 5 & 4,3 & $\begin{array}{c}3,92 \\
(-6,08) \\
\end{array}$ \\
\hline Libia & $0,8(-9,2)$ & 2,57 & 2,19 & - & 1,7 & $\begin{array}{c}2,32 \\
(-7,68) \\
\end{array}$ \\
\hline Argelia & $\begin{array}{c}2,15 \\
(-7,85)\end{array}$ & 4,75 & 3.5 & 5,1 & 3.5 & $\begin{array}{c}3,72 \\
(-6,28)\end{array}$ \\
\hline Egipto & $\begin{array}{c}1,43 \\
(-8,57)\end{array}$ & 3.7 & 3,36 & 3,6 & 3.5 & $\begin{array}{c}2,64 \\
(-7,36)\end{array}$ \\
\hline
\end{tabular}

Fuente: Elaboración propia a través de los principales indices de democracia.

Una vez explicada la metodología utilizada en ciertos datos del marco teórico ajenos a la revisión bibliográfica, vamos a exponer la metodología utilizada en el cuerpo de la investigación. En primer lugar, vamos a utilizar datos primarios, al estudiar si ciertos elementos aparecen en las páginas web de los parlamentos de diferentes países. Esos elementos los definiremos como variables, y les daremos el valor 1 en el caso de que aparezcan en un pais y el valor o en el caso de que no aparezcan. Los datos serán recogidos mediante la revisión documental de las páginas web y serán recopilados mediante una tabla.

Las variables las definiremos de manera comparada, haciendo un análisis de contenido de la página del Senado español de carácter cualitativo. Todos los elementos que encontremos en esa página serán convertidos en dimensiones de análisis y variables a estudiar. El análisis de contenido comparado nos permitirá observar cómo varían esas variables según las páginas de los diferentes países. Las variables que hemos definido constan en la Tabla 3. 


\section{Carlos José Aguilar García}

Tabla. 3 Variables definidas

\begin{tabular}{|c|c|}
\hline $\begin{array}{c}\text { Variables y } \\
\text { Dimensiones }\end{array}$ & Descripción \\
\hline Doo & Dimensión: gran apartado de actividad parlamentaria \\
\hline V01 & Apartado: una ventana de actualidad \\
\hline V010 & Leyes aprobadas \\
\hline V011 & Directos del parlamento \\
\hline V012 & Prensa y medios sobre senado \\
\hline Vo2 & Apartado pleno \\
\hline Vo3 & Diputación permanente/ órgano correspondiente en el país \\
\hline Vo4 & Comisiones y ponencias \\
\hline Vo5 & Publicaciones oficiales \\
\hline Vo6 & Relaciones Internacionales \\
\hline D10 & Dimensión: gran apartado donde están otros apartados: Composición y organización \\
\hline SD11 & Subdimensión; apartado sobre senadores \\
\hline V110 & Actividad parlamentaria del parlamentario \\
\hline V111 & Biografía del parlamentario \\
\hline$V_{112}$ & Declaraciones de bienes patrimonios y renta de los parlamentarios \\
\hline V113 & Otras legislaturas en el parlamento \\
\hline V114 & Composición parlamento \\
\hline V115 & Estatuto de funcionamiento \\
\hline$V_{116}$ & Parlamentarios en todas las legislaturas incluso antes de el actual régimen \\
\hline V117 & Régimen económico \\
\hline V118 & Protección social \\
\hline V119 & Sistema electoral \\
\hline V1110 & Resultados electorales \\
\hline SD12 & Subdimensión: apartado sobre grupos parlamentarios \\
\hline V120 & Apartado de cada grupo parlamentario \\
\hline$V_{121}$ & Fecha de constitución del grupo \\
\hline$V_{122}$ & Composición del grupo \\
\hline V123 & Iniciativas del grupo \\
\hline$V_{124}$ & Partidos políticos que lo componen \\
\hline V125 & Redes sociales del partido grupo \\
\hline$V_{126}$ & subvenciones grupos parlamentarios \\
\hline$V_{127}$ & Funciones grupos parlamentarios \\
\hline$V_{128}$ & Grupos parlamentarios en la historia del parlamento \\
\hline SD13 & Subdimensión: órganos del parlamento \\
\hline V131 & Presidentes desde la institución de la cámara \\
\hline$V_{132}$ & Composición órganos \\
\hline$V_{133}$ & Sesiones actividad órganos \\
\hline SD14 & Subdimensión: administración parlamentaria \\
\hline V141 & Organigrama \\
\hline $\mathrm{V}_{142}$ & Normas \\
\hline D-V2 & Dimensión: datos culturales sobre la cámara \\
\hline D-V3. & Dimensión: búsqueda temática \\
\hline
\end{tabular}

Fuente: Elaboración propia a través de la página web del Senado español ${ }^{4}$

4 Página oficial Senado de España: http://www.senado.es/ 


\section{La transparencia en las páginas web de los parlamentos ...}

En esta tabla agrupamos las variables en diferentes dimensiones de análisis (D) y subdimensiones (SD), además de exponer su contenido. Los elementos marcados en rojo y en negrita hacen referencia a las dimensiones, dentro de ellas encontramos diferentes variables (V). En algunos casos hemos agrupados las variables en subdimensiones dentro de una dimensión de análisis, como es el caso de la dimensión composición y organización, donde encontramos cuatro subdimensiones, marcadas en cursiva y en verde. Cada subdimensión recoge en su interior diferentes variables. En las dos últimas dimensiones hemos utilizado el elemento $\mathrm{D}-\mathrm{V}$ ya que hacen referencia a la transparencia colaborativa ${ }^{5}$, y esta tiene poca presencia en los regímenes no democráticos, por lo que usaremos una única variable amplia, para cada dimensión, que incluirá todos los elementos de estas dimensiones.

Los siguientes apartados del trabajo versarán sobre el análisis de cada una de estas variables en las diferentes páginas web de una de las cámaras de Marruecos, Argelia y Túnez. Para el análisis de contenido de estas páginas hemos utilizado el traductor de Google que de manera directa convierte las páginas del árabe a cualquier idioma. Hemos preferido esta técnica a leerlas según la propia traducción de los países por si sus páginas web ocultan información cuando son traducidas. En el caso de haber tenido que acudir a un documento pdf dentro de la página web, hemos utilizado diferentes traductores para conocer el contenido, por ejemplo, si algún enlace de la página del Senado marroqui nos dirigía a un pdf de la Constitución de Marruecos en árabe.

A continuación, expondremos el desarrollo de este análisis sobre el Senado de Marruecos, el Senado de Argelia y el Congreso de Túnez (para el caso tunecino simplemente cambiaremos las variables que se refieren a senadores para referirnos a diputados).

\section{TRANSPARENCIA ACTIVA: LA ACTUALIDAD PARLAMENTARIA}

La actualidad parlamentaria es una de las dimensiones de análisis que mejor evidencia la transparencia política, en ella encontramos todo tipo de variables que lo muestran, como la presencia de los medios en las cámaras o las decisiones tomadas por los parlamentos. Elegimos esta dimensión como forma de transparencia activa por su intento de difundir la actividad de la cámara a la ciudadanía, lo que evidencia una actividad a favor de la formación del ciudadano. A continuación incluimos la tabla en la que se reflejan las variables de este apartado y su aparición o no en los diferentes países.

\footnotetext{
5 Esto queda expuesto en el apartado de Transparencia Colaborativa, pero a modo de aclaración, incluimos estas dimensiones en este tipo de transparencia porque permiten al ciudadano interconectar ideas y mantener un diálogo con la página de la administración para llegar a conclusiones e información que quizás no esperaba
} 


\section{Carlos José Aguilar García}

Tabla 4. Variables de actualidad parlamentaria por país

\begin{tabular}{|l|l|c|c|c|c|}
\hline \multicolumn{2}{|c|}{ Variables } & España & Túnez & Marruecos & Argelia \\
\hline Do0 & $\begin{array}{l}\text { Dimensión: gran apartado de actividad } \\
\text { parlamentaria }\end{array}$ & $\mathrm{SI}$ & $\mathrm{SI}$ & $\mathrm{SI}$ & $\mathrm{SI}$ \\
\hline V01 & Apartado: una ventana de actualidad & $\mathrm{SI}$ & $\mathrm{SI}$ & $\mathrm{SI}$ & $\mathrm{SI}$ \\
\hline V010 & Leyes aprobadas & $\mathrm{SI}$ & $\mathrm{SI}$ & $\mathrm{SI}$ & $\mathrm{SI}$ \\
\hline V011 & Directos del parlamento & $\mathrm{SI}$ & $\mathrm{SI}$ & $\mathrm{NO}$ & $\mathrm{SI}$ \\
\hline V012 & Prensa y medios sobre senado & $\mathrm{SI}$ & $\mathrm{NO}$ & $\mathrm{NO}$ & $\mathrm{NO}$ \\
\hline V02 & Apartado pleno & $\mathrm{SI}$ & $\mathrm{SI}$ & $\mathrm{NO}$ & $\mathrm{SI}$ \\
\hline V03 & $\begin{array}{l}\text { Diputación } \\
\text { correspondiente en el pais }\end{array}$ & $\mathrm{SI}$ & $\mathrm{No}$ & $\mathrm{No}$ & $\mathrm{SI}$ \\
\hline V04 & Comisiones y ponencias & $\mathrm{SI}$ & $\mathrm{SI}$ & $\mathrm{SI}$ & $\mathrm{SI}$ \\
\hline V05 & Publicaciones oficiales & $\mathrm{SI}$ & $\mathrm{SI}$ & $\mathrm{SI}$ & $\mathrm{SI}$ \\
\hline V06 & Relaciones Internacionales & $\mathrm{SI}$ & $\mathrm{SI}$ & $\mathrm{SI}$ & $\mathrm{SI}$ \\
\hline
\end{tabular}

Fuente: elaboración propia a través de las páginas web de los parlamentos de los diferentes países

La dimensión actividad parlamentaria, que encabeza la lista de variables, aparece completa en todas, ya que en mayor o menor medida existe un apartado en sus páginas web que las acoja. Debemos destacar que todos los regímenes dentro de este apartado recogen una ventana o enlace a la actualidad parlamentaria. Esta variable tiene en común, en todos los países, que no se refiere solo a la actividad legislativa del parlamento, sino que es un apartado amplio, en el que se recoge si algunos grupos internacionales han visitado el parlamento o si se han reunido algunos grupos parlamentarios aparte del pleno. La importancia de esta variable dependerá de si los países realmente introducen todo lo que ocurre en los edificios de sus parlamentos o no.

Todos, a su vez, tienen la variable Vo10, que recoge las leyes que se van aprobando, lo que era de esperar, ya que las cámaras legislativas tienen como objeto aprobar las leyes del país en la mayoría de los casos, luego no tendría sentido su no aparición. A partir de la siguiente variable empezarán las diferencias, que casualmente marcarán su mayor grado en Marruecos y no en Argelia, siendo este último menos democrático.

\footnotetext{
6 Página oficial Senado de Marruecos: http://www.maroc.ma/es/content/parlamento

Página oficial del Congreso de Túnez:: http://www.arp.tn/site/main/AR/index.jsp

Página oficial del Senado de Argelia: http://www.majliselouma.dz/index.php/ar/
} 


\section{La transparencia en las páginas web de los parlamentos ...}

Marruecos en la página web de su senado carece de un directo que emita los plenos desde la página web, y de un apartado dedicado expresamente a los plenos. Tampoco tiene un apartado sobre prensa y medios que traten temas del Senado, lo que lo convierte en la cámara más opaca en esta dimensión de las cuatro seleccionadas. Argelia, por su parte, carece de un apartado de prensa, pero sí tiene uno en el que conste la realidad de los plenos y sí tiene un apartado de directos, al igual que Túnez. Como podemos observar, es algo común a los tres países magrebies la ausencia de un apartado sobre prensa de la cámara, lo que evidencia la necesidad de ahondar más en la transparencia y la rendición de cuentas ante el mundo privado.

Por último, debemos destacar que todos gozan de un apartado de Relaciones Internacionales, en el que se reflejan las visitas y viajes a otros países de los diputados o senadores, los acuerdos de apoyo o colaboración o incluso las visitas de formación que puedan hacer los parlamentarios. También gozan de un espacio para desglosar las comisiones y ponencias, lo que es bastante sorprendente, y otro de publicaciones oficiales.

En definitiva, en este apartado podemos observar cómo el país que más adolece de transparencia no es el más autoritario, sino el segundo en la cola Marruecos. Por otro lado, Argelia y Túnez obtienen unos resultados similares, lo que podría marcar la diferencia sería un estudio más centrado en la exposición del contenido de cada apartado, en el cual quizás veríamos profundas diferencias entre los regímenes autoritarios y la democracia defectiva.

\section{TRANSPARENCIA PASIVA: COMPOSICIÓN Y ORGANIZACIÓN}

La composición y la organización de las cámaras son un elemento de vital importancia para el funcionamiento democrático de las instituciones. Conocer los mecanismos por los que se eligen miembros o se toman decisiones estará dentro de este apartado. Este refleja una forma de transparencia pasiva, que se centra en dar acceso a la ciudadanía, como una forma de ejercer la rendición de cuentas más técnicas y no tan enfocada al fomento de la participación. Para abarcar una materia tan amplia hemos dividido esta dimensión en 4: los parlamentarios (SD11 Senadores o Diputados), los grupos parlamentarios (SD12), los órganos del Senado o Congreso (SD13) y la Administración Parlamentaria (SD14). 


\section{Carlos José Aguilar García}

Tabla 5. Variables de composición y organización por país

\begin{tabular}{|c|c|c|c|c|c|}
\hline \multicolumn{2}{|c|}{ Variables } & \multirow{2}{*}{\begin{tabular}{|c|} 
España \\
SI
\end{tabular}} & \multirow{2}{*}{\begin{tabular}{|c|} 
Túnez \\
SI \\
\end{tabular}} & \multirow{2}{*}{$\begin{array}{c}\text { Marruecos } \\
\mathrm{SI}\end{array}$} & \multirow{2}{*}{\begin{tabular}{|c} 
Argelia \\
SI
\end{tabular}} \\
\hline D10 & $\begin{array}{l}\text { Dimensión: gran apartado donde están otros apartados: } \\
\text { Composición y organización }\end{array}$ & & & & \\
\hline SD11 & Subdimensión; apartado sobre senadores & $\mathrm{SI}$ & $\mathrm{SI}$ & NO & $\mathrm{SI}$ \\
\hline$V_{110}$ & Actividad parlamentaria del parlamentario & $\mathrm{SI}$ & $\mathrm{NO}$ & $\mathrm{NO}$ & $\mathrm{NO}$ \\
\hline$V_{111}$ & Biografia del parlamentario & $\mathrm{SI}$ & $\mathrm{NO}$ & NO & NO \\
\hline V112 & $\begin{array}{l}\text { Declaraciones de bienes patrimonios y renta de los } \\
\text { parlamentarios }\end{array}$ & SI & NO & NO & NO \\
\hline V113 & Otras legislaturas en el parlamento & $\mathrm{SI}$ & NO & NO & NO \\
\hline V114 & Composición parlamento & $\mathrm{SI}$ & $\mathrm{SI}$ & $\mathrm{SI}$ & $\mathrm{SI}$ \\
\hline V115 & Estatuto de funcionamiento & $\mathrm{SI}$ & NO & $\mathrm{NO}$ & $\mathrm{SI}$ \\
\hline$V_{116}$ & $\begin{array}{l}\text { Parlamentarios en todas las legislaturas incluso antes de } \\
\text { el actual régimen }\end{array}$ & $\mathrm{SI}$ & $\mathrm{SI}$ & NO & NO \\
\hline$V_{117}$ & Régimen económico & $\mathrm{SI}$ & NO & NO & NO \\
\hline V118 & Protección social & $\mathrm{SI}$ & NO & NO & NO \\
\hline V119 & Sistema electoral & SI & $\mathrm{SI}$ & $\mathrm{NO}$ & $\mathrm{NO}$ \\
\hline$V_{1110}$ & Resultados electorales & $\mathrm{SI}$ & NO & NO & NO \\
\hline SD12 & Subdimensión: apartado sobre grupos parlamentarios & $\mathrm{SI}$ & $\mathrm{SI}$ & $\mathrm{SI}$ & $\mathrm{SI}-\mathrm{NO}$ \\
\hline V120 & Apartado de cada grupo parlamentario & $\mathrm{SI}$ & $\mathrm{SI}$ & $\mathrm{SI}$ & $\mathrm{SI}$ \\
\hline $\mathrm{V}_{121}$ & Fecha de constitución del grupo & SI & NO & NO & NO \\
\hline V122 & Composición del grupo & $\mathrm{SI}$ & SI & $\mathrm{SI}$ & $\mathrm{SI}$ \\
\hline V123 & Iniciativas del grupo & $\mathrm{SI}$ & NO & $\mathrm{NO}$ & $\mathrm{NO}$ \\
\hline V124 & Partidos políticos que lo componen & $\mathrm{SI}$ & $\mathrm{SI}$ & NO & NO \\
\hline V125 & Redes sociales del partido grupo & SI & NO & NO & NO \\
\hline$V_{126}$ & subvenciones grupos parlamentarios & $\mathrm{SI}$ & NO & NO & NO \\
\hline V127 & Funciones grupos parlamentarios & $\mathrm{SI}$ & NO & NO & NO \\
\hline$V_{128}$ & Grupos parlamentarios en la historia del parlamento & $\mathrm{SI}$ & NO & NO & NO \\
\hline SD13 & Subdimensión: órganos del parlamento & $\mathrm{SI}$ & $\mathrm{SI}$ & $\mathrm{SI}$ & $\mathrm{SI}$ \\
\hline V131 & Presidentes desde la institución de la cámara & $\mathrm{SI}$ & $\mathrm{SI}$ & NO & NO \\
\hline V132 & Composición órganos & $\mathrm{SI}$ & $\mathrm{SI}$ & $\mathrm{SI}$ & $\mathrm{SI}$ \\
\hline V133 & Sesiones actividad órganos & $\mathrm{SI}$ & $\mathrm{SI}$ & $\mathrm{SI}$ & $\mathrm{SI}$ \\
\hline SD14 & Subdimensión: administración parlamentaria & $\mathrm{SI}$ & SI & $\mathrm{NO}$ & $\mathrm{NO}$ \\
\hline$V_{141}$ & Organigrama & SI & NO & NO & $\mathrm{NO}$ \\
\hline$V_{142}$ & Normas & $\mathrm{SI}$ & $\mathrm{SI}$ & $\mathrm{SI}$ & $\mathrm{SI}$ \\
\hline
\end{tabular}

Fuente: elaboración propia a través de las páginas web de los parlamentos de los diferentes países 


\section{La transparencia en las páginas web de los parlamentos ...}

Dentro de SD11, referida a los parlamentarios, encontramos una mayor opacidad que en el anterior apartado y una notable diferencia entre Túnez y los otros dos, siendo además y de nuevo Marruecos el país más opaco. Todos menos el Senado marroquí incluyen un apartado sobre los parlamentarios, esto ya plantea un primer obstáculo para encontrar la información. Lo primero que observamos es que ningún país incluye datos sobre la vida personal de los parlamentarios, ni de su actividad, quedando vacias las variables: V110, que hace referencia a la actividad parlamentaria, V111 que identifica si existen apartados que recogen la biografía de los parlamentarios, donde irían incluidos aquellos empleos que ha realizado a lo largo de su vida, V112, donde se recogen las declaraciones de bienes, patrimonios y rentas de los parlamentarios, siendo esta la variable más económica, pudiendo habernos permitido analizar el nivel de vida de los parlamentarios de los diferentes países si la hubiesen recogido y $V_{113}$, donde podríamos observar otras legislaturas en las que habría participado el parlamentario. Siendo así, totalmente oculta la vida privada de los parlamentarios, sus rentas, sus propuestas de ley o si han sido miembros del parlamento con anterioridad.

En las variables; V114, que versa sobre la composición del parlamento, V115 sobre el estatuto de funcionamiento de cada cámara que estudiamos, V116, que recoge un histórico de todos los parlamentarios, y $V_{11}$, que hace referencia al sistema electoral, encontramos algunas diferencias. Túnez es el único país que incluye un apartado histórico de los senadores que han compuesto la cámara, mientras que Argelia es el único que tiene colgado su estatuto de funcionamiento. Los tres países tienen colgada la composición de su cámara, pero tan solo Túnez expone su sistema electoral.

V117, sobre el régimen económico, V118, sobre la protección social de los parlamentarios, y $\vee 1110$, sobre los resultados electorales, destacan por su ausencia en todos los países. En ninguno se recogen los resultados electorales según el escrutinio de votos, tan solo podemos intuirlo contando el número de diputados por grupos. Tampoco incluyen el régimen económico de los parlamentarios ni su protección social. Esta subdimensión repite la misma tendencia que la del primer apartado, con la única diferencia de que Túnez empieza a repuntar.

En el ámbito de los grupos parlamentarios (SD12) debemos destacar que todos tienen esta pestaña, pero que Argelia, en octubre cuando intentamos recuperar la información, no nos permitía acceder a la pestaña de los grupos, por lo que tuvimos que entrar desde la de membresía y desde ahí indagar entre los miembros y la información que aparecía, es por eso por lo que a la variable le concedemos el valor Sí y No a la vez.

Esta subdimensión es quizás en la que más opacidades encontramos. Con respecto a las variables, no hemos encontrado ningún tipo de enlace sobre las que hacen referencia a la siguiente información: la fecha de constitución de los grupos parlamentarios ( $\left.V_{121}\right)$, las iniciativas legislativas de los grupos (V123), las redes 


\section{Carlos José Aguilar García}

sociales de estos ( $V_{125}$ ), si es que tuviesen, si reciben algún tipo de subvención ( $\left.V_{126}\right)$, las funciones de las que gozan ( $\left.V_{127}\right)$, y los grupos que ha habido a lo largo de la historia de sus parlamentarismos (V128).

Las únicas variables en las que algunos de los paises magrebies seleccionados tienen información son la $V_{122}$, que se refiere a la composición de los grupos parlamentarios en número de personas. Algunos países aportan más información y otros menos, en este caso dependiendo de su nivel de democratización, según la tipología utilizada al inicio. Sin embargo, hemos señalado esta variable en todos porque al menos podemos saber qué personas lo componen. En la V124, que se refiere a los partidos políticos que componen el grupo parlamentario, de nuevo destaca Túnez, que es el único que aporta de manera clara esta información.

En la subdimensión referente a los órganos del Senado o Congreso (SD13) encontramos los niveles más altos de transparencia. Aquí, todos tienen un apartado en materia de órganos de la cámara, V132, de la composición de los mismos V133, y de las sesiones y actividad de los órganos, V13. Sin embargo, en el caso de V131, sobre la información del Presidente, vuelve a destacar Túnez, siendo el único con una pestaña dedicada a él en su página web y consolidándose como el país más transparente.

Por último, tan solo Túnez tiene un apartado expreso haciendo referencia a la administración parlamentaria (SD14), y en ella recoge el organigrama y las normas de la cámara (V141 y V142). Sin embargo, aunque Túnez sea el único con todas las variables de esta sección, el resto de países sí incluyen las normas de la cámara.

\section{TRANSPARENCIA COLABORATIVA: CULTURA Y BÚSQUEDA TEMÁTICA}

Este último apartado lo hemos definido como de transparencia colaborativa por su intención de interacción con el ciudadano. Cuando nos referimos a un apartado de búsqueda en la página web no solo estamos dando una forma más sencilla al ciudadano de buscar la información que precisa, sino que estamos fomentando su imaginación en lo que espera de la administración. Cuando una página web pública tiene un buscador desarrollado, este tiene la capacidad de buscar no solo por títulos, sino también por palabras y temas, permitiendo a los ciudadanos interconectar ideas y mantener un diálogo con la página de la administración para llegar a conclusiones e información que quizás no esperaba, adquiriendo el grado de colaborativa

También hemos incluido en la transparencia colaborativa la dimensión de la cultura porque, aunque no se refiera estrictamente a lo establecido a nivel teórico, en un contexto no democrático los parámetros han de ser diferentes. Los conceptos teóricos de la transparencia, en los casos seleccionados, se centran en modelos democráticos es por ello por lo que hay que hacer ciertas apreciaciones para los regímenes híbridos. La cultura en las páginas oficiales es un elemento colaborativo 


\section{La transparencia en las páginas web de los parlamentos ...}

de transparencia en tanto en cuanto la ciudadanía de un régimen autoritario no tiene las facilidades que existen en un régimen democrático para acceder a ella, y la aparición de la cultura en una página de una administración no democrática no tiene solo un carácter activo, sino colaborativo en su relación con el ciudadano. Por esta razón, incluimos aquí estas dos variables.

Tabla 6. Variables de cultura y búsqueda temática por país

\begin{tabular}{|l|l|c|c|c|c|}
\hline \multicolumn{2}{|c|}{ Variables } & España & Túnez & Marruecos & Argelia \\
\hline D-V2 & Dimensión: datos culturales sobre la cámara & $\mathrm{SI}$ & $\mathrm{NO}$ & $\mathrm{NO}$ & $\mathrm{SI}$ \\
\hline D-V3 & Dimensión: búsqueda temática & $\mathrm{SI}$ & $\mathrm{NO}$ & $\mathrm{NO}$ & $\mathrm{NO}$ \\
\hline
\end{tabular}

Fuente: elaboración propia a través de las páginas web de los parlamentos de los diferentes países.

Como podemos observar, y para sorpresa de nuestro estudio, tan solo Argelia tiene una de estas variables y es la de la cultura, ninguno de los países tiene un buscador oficial y (como ya hemos señalado) solo uno tiene información cultural. Este apartado evidencia que la relación entre transparencia colaborativa y régimen híbrido en el Magreb tiene aún mucho que avanzar.

\section{CONCLUSIONES}

Al inicio del trabajo señalamos que relacionaríamos tres de las dimensiones de Villoria con los tipos de transparencia y veríamos su impacto sobre cada país. De esta manera, tenemos una transparencia pasiva de carácter técnico centrada en la rendición de cuentas, una transparencia activa, de carácter político, basada en el gobierno participativo y una transparencia colaborativa fundamentada en la promoción de civismo. Las dimensiones de análisis, a nuestro juicio, han sido acertadas para analizar cada una de estas transparencias en los diferentes países y nos ponen de manifiesto diferentes cuestiones, que queremos evidenciar en la siguiente tabla, que incorpora el número y la proporción de variables que han cumplido cada país por tipo de transparencia.

Con estos resultados podemos concluir que si existe una clara diferencia entre los regímenes autoritarios y los regímenes democráticos defectivos a la hora de tratar la transparencia pasiva. Esta transparencia, que es de carácter más técnico, es más fácil de alcanzar para los regímenes cuyas instituciones tienden a asimilarse más a las de un régimen completamente democrático (lo primero que suelen hacer los regímenes es copiar las instituciones), por lo que es normal que la democracia defectiva sea la más transparente en su carácter técnico. 


\section{Carlos José Aguilar García}

Tabla 7. Tipos de transparencia total por país

\begin{tabular}{|l|c|c|c|c|}
\hline \multicolumn{1}{|c|}{$\begin{array}{c}\text { Tipo de } \\
\text { Transparencia }\end{array}$} & España & Túnez & Marruecos & Argelia \\
\hline $\begin{array}{l}\text { Transparencia } \\
\text { pasiva }\end{array}$ & $100 \%$ & $50 \%$ & $30 \%$ & $36,7 \%$ \\
\cline { 2 - 5 } & 30 & 15 & 9 & 11 \\
\hline $\begin{array}{l}\text { Transparencia } \\
\text { activa }\end{array}$ & $100 \%$ & $88,9 \%$ & $66,7 \%$ & $88,9 \%$ \\
\cline { 2 - 5 } & 9 & 8 & 6 & 8 \\
\hline $\begin{array}{l}\text { Transparencia } \\
\text { colaborativa }\end{array}$ & $100 \%$ & $0 \%$ & $0 \%$ & $50 \%$ \\
\cline { 2 - 5 } & 2 & 0 & 0 & 1 \\
\cline { 2 - 5 } $\begin{array}{l}\text { Transparencia } \\
\text { total }\end{array}$ & 41 & 23 & 15 & 20 \\
\hline
\end{tabular}

Fuente: elaboración propia a través de las páginas web de los parlamentos de los diferentes países.

En la transparencia activa la realidad es sorprendente, la democracia defectiva y el autoritarismo restrictivo tienen prácticamente el mismo número de variables atendidas que la democracia plena. Esto puede llevarnos a varias conclusiones, en primer lugar, que es posible que el régimen argelino en el ámbito formal quiera dar una apariencia de democracia, con unos altos niveles de transparencia activa formal, quizás un análisis de contenido más profundo hubiera sido más pertinente para conocer la realidad de esta igualdad formal. Sorprende además, en segundo lugar, como aquí, de nuevo, es sobrepasado el régimen marroquí por otro régimen más autoritario en sus niveles de transparencia. En tercer lugar, vemos cómo Túnez en su deriva democrática tiene cada vez más variables formales en el ámbito de la transparencia activa cercanas a una democracia plena como España.

Por último, debemos señalar que los resultados obtenidos con las dimensiones referentes a la transparencia colaborativa no pueden ser concluyentes por el reducido número de variables que contienen. Sin embargo, es destacable que Argelia sea el único de los tres países que recoge un apartado sobre cultura.

En conclusión, podemos decir que al menos en el ámbito formal, nuestra hipótesis no se cumple, ya que los regímenes no siguen una relación lineal entre transparencia en los portales de sus parlamentos y nivel de democracia, Marruecos siendo un autoritarismo pluralista, es el que menos transparencia refleja en su 


\section{La transparencia en las páginas web de los parlamentos ...}

página web, y Argelia se aproxima en muchas ocasiones al régimen tunecino, luego podemos concluir que no existe relación entre los niveles de transparencia de sus administraciones parlamentarias en la red y su capacidad democrática. Esto puede ser fruto de tres factores: en primer lugar, que las categorías de regímenes utilizados en este estudio no han recogido de manera correcta la información sobre transparencia electrónica, por lo que no cuadra una mayor transparencia en las páginas web con mayores niveles de democracia según nuestra tipología de regímenes. En segundo lugar, que no haya sido adecuado el uso de España como modelo de referencia, y por eso hemos encontrado ciertas contradicciones, como la de la transparencia colaborativa. En último lugar, podría ocurrir que al estudiar el contenido de cada una de las pestañas que hemos recogido el análisis fuera diferente, y que países autoritarios como Argelia quisieran dar una cara formalmente más democrática en su web que no se tradujera a la hora de buscar la información dentro de cada una de sus pestañas.

En definitiva, estas conclusiones nos permiten plantear futuros trabajos que se podrian abordar: por un lado, el estudio de las páginas web de estos países con otro país como referencia, que generara otras dimensiones de análisis y otras variables, y, por otro lado, el estudio del contenido integro de cada una de las pestañas que nosotros hemos recogido de manera formal. Asi podriamos comparar ambos resultados y conocer qué países distorsionan su imagen en la página web de su parlamento con respecto al contenido de la misma 


\section{Carlos José Aguilar García}

\section{BIBLIOGRAFÍA.}

Arendt, H. 2006. Los origenes del totalitarismo. Alianza: Madrid.

Camau, M. 1992. "Poder y legitimidad en el Magreb", en López García, B, EspañaMagreb Siglo XXI. Mapfre: Madrid.

Canales Aliende, J. M., \& Romero-Tarín, A. 2017. "Algunas consideraciones sobre la transparencia pública y el buen gobierno". Revista Española de Transparencia, 5: $115-127$.

Cerillo, Agustí. 2011. "Transparencia administrativa y lucha contra la corrupción en la administración local", Anuario de Gobierno local 2011, 277-313.

Curto-Rodriguez, R. 2019. “¿Qué información relacionada con la publicidad activa contienen los portales de datos abiertos autonómicos españoles? Un análisis detallado tras cuatro años de su eclosión". Revista española de la transparencia, 9: $177-198$.

Delgado Morales, F. 2015. "De qué hablamos cuando hablamos de transparencia". Revista española de la transparencia, 1: 23-30.

Diamond, L., J.J. Linz y S.M. Lipset. 1995. Politics in developing Countries, Boulder, CO, Lynne Rienner Publisher

Entelis, J. P. 2008. "Entre los deseos democráticos y las tentaciones autoritarias en el Magreb central", en el Magreb realidades nacionales y dinámicas regionales. Sintesis: Madrid.

Fernández, Y., Fernández, J.M. y Rodríguez, A. 2008. "Modernización de la Gestión Pública: Necesidad, incidencias, límites y críticas". PECVNIA, 6: 75-105

Fuentes, Juan Francisco. 2006. "Totalitarismo: origen y evolución de un concepto clave", Revista de Estudios Políticas, 134: 195-218.

Garcia-Garcia, J., \& Curto-Rodriguez, R. 2018. Divulgación de información pública de las Comunidades autónomas españolas (2013-2017): Portal de datos abiertos, portal de transparencia y web institucional. El profesional de la información, 27(5): 1051-1060.

Garcia-García, J. y Curto-Rodríguez, R. 2019. "El ejercicio de la rendición de cuentas mediante portales de datos abiertos en las comunidades autónomas españolas". IDP. Revista de Internet, Derecho y Politica, 29: 1-15 DOI: http://doi.org/10.7238/idp.voi29.3182 


\section{La transparencia en las páginas web de los parlamentos ...}

Liebert, U. 1988. "Parlamento y consolidación de la democracia en la Europa del sur". Revista Española de Investigaciones Sociológicas. 42: 93-136

Linz, J.J. 1990. "Transiciones a la democracia". Revista Española de Investigaciones sociológicas. 51: 7-34.

Máiz, R. 2006. "Deliberación e inclusión en la democracia republicana". Revista Española Investigaciones Sociológicas. 113: 11-47.

Montero, J.R. \& Morlino, L. 1993. "Legitimidad y democracia en el Sur de Europa". Revista Española de Investigaciones sociológicas. 64: 7-40.

Morlino, L. 2015. "Transiciones democráticas: entre teóricas y análisis empírico", Revista Española de Ciencia Política. 39: 17-42.

Morlino, L. 1986. "Consolidación democrática. Definición, modelos, hipótesis". Revista española de investigaciones sociológicas. 35: 7-61.

Parejo, M. A. 2010. Entre el autoritarismo y la democracia, los procesos electorales en el Magreb. Bellaterra, Madrid.

Szmolka, I. 2010. "Los regímenes políticos híbridos: democracias y autoritarismos con adjetivos, su conceptualización, categorización y operacionalización dentro de la tipología de regímenes políticos", Revistas de Estudios Politicos, 147: 103135 .

Szmolka, I. 2011. "Democracias y autoritarismos con adjetivo: la clasificación de los países árabes dentro de una tipología general de regímenes políticos", Revista Española de Ciencia Política, 26: 11-62.

Szmolka, I. 2019. "Una propuesta de tipología de sistemas de partidos para regímenes democráticos y autoritarios pluralistas. Su aplicación a los países del Magreb". Revista Española de Investigaciones Sociológicas, 168: 93-110

O'Donnell, G. y Schmitter, P.C. 1994. Transiciones desde un gobierno autoritario. Barcelona: Paidós.

Tudela, J. 2012. "Parlamento y Derechos Fundamentales". Revista Advocatus, 10: 207-226.

Villoria, M. 2013. "El gobierno abierto como subsistema de políticas: Una evaluación desde el institucionalismo discursivo". En Ramírez-Alujas, A., Hofmann, A., y Bojórquez Pereznieto, J.A. (Coords.): La promesa del gobierno abierto, pg 69-101.

Villoria, M. 2018. "La transparencia, la imparcialidad y la calidad de la democracia", Ilemata, Revista Internacional de éticas aplicadas, 27: 1-16. 
